\title{
MÉTIERS DE LA COMMUNICATION ET PROCESSUS DE LÉGITIMATION : LE « COMMUNICATEUR-SYMPTÔME »
}

\author{
Pierre de Saint-Georges ${ }^{1}$
}

L'hypothèse sous-tendant la réflexion proposée ici est que les pratiques professionnelles des « communicateurs d'organisation » révèlent la nature des rapports sociaux que tendent à légitimer ces organisations. Précisons d'emblée que le terme d'organisation est pris dans un sens générique. Il désigne aussi bien les entreprises que les administrations ou les associations. Et la notion de «communicateurs» renvoie quant à elle aux praticiens des métiers spécifiques de la communication. De ce point de vue, « communiquer », c'est d'une manière ou d'une autre intervenir sur (mais aussi dans) un système de comportements et d'opinions afin d'y induire du changement (d'information, d'opinion, de comportement ...).

Mais on se trouve en fait directement en présence de deux registres de réflexion régulièrement confondus utilisant chacun le terme « communication » à leur manière. Le premier de ces deux registres étudie les phénomènes et les dispositifs constitutifs de la vie en société elle-même (et singulièrement ici de la vie des organisations). L'organisation y est lue comme une forme sociale mise en dynamique

1 Professeur à l'Université Catholique de Louvain et aux Facultés Universitaires Saint-Louis à Bruxelles. Co-fondateur du Laboratoire d'analyse des systèmes de communication des organisations (UCL - LASCO).

Recherches en communication, $\mathrm{n}^{\circ} 25$ (2006). 
par ses acteurs dans un contexte social donné ${ }^{1}$. Elle est donc directement appréhendée comme un système de communication (comme d'autres y verraient un système de pouvoir). Elle ne lui préexiste pas. Le second registre porte quant à lui sur les actions d'une catégorie de professionnels : les «communicateurs ». Il s'agit de comprendre, mais aussi de chercher à optimiser, les stratégies qu'ils mènent à l'intérieur comme vers l'extérieur de l'organisation à laquelle ils sont contractuellement liés, les buts qu'ils se fixent, les moyens et les méthodes qu'ils mettent en œuvre.

Assimiler ces deux niveaux d'analyse en confondant le second avec le premier (ce qui n'est malheureusement pas exceptionnel) est à nos yeux se condamner à une approche métonymique de la communication qui rend la notion de communication particulièrement polysémique, voire ambiguë. C'est confondre « communiquer» et «faire de la communication ». C'est ne pas distinguer la communication organisationnelle (qui se réfère à la dynamique communicationnelle nécessairement constitutive de toute organisation) de la communication d'organisation (forcément instrumentale dans la mesure où il s'agit de concevoir et de mettre en œuvre les stratégies de communication décidées par le management).

Mais bien sûr, en tout cas du point de vue du cadre théorique de la pragmatique de la communication notamment (Bateson, Watzlawick, Goffman, Winkin, etc), sans que cela ne supprime la distinction que nous venons de poser, «faire de la communication » est évidemment aussi se comporter, donc communiquer. «Faire de la communication » est de ce fait une classe spécifique de comportements. Les missions et les actions des communicateurs apparaissent alors dans ce cadre comme des analyseurs particulièrement sensibles des rapports de communication au sens plein, car elles ont pour fonction d'agir sur la communication, c'est-à-dire sur des comportements, des opinions et sur ce qui les régule. Nous allons essayer de voir pourquoi.

1 Voir par exemple à ce sujet Duterme Cl. (2002), La communication interne en entreprise, Bruxelles, De Boeck; Pirson J., (1999), De l'analyse stratégique à l'analyse pragmatique, in Recherches sociologiques, 3, pp 131-146; Salamanca Avila, L., (2003), Piloter le changement dans les organisations, Louvain-la-Neuve, Presses universitaires de Louvain. 


\section{Communiquer sans « communicateurs »}

Plusieurs dimensions interviennent dans la définition de l'identité d'une organisation que nous prenons ici, un peu arbitrairement, comme point de départ de l'analyse du rôle et de la position des communicateurs. Une dimension d'ordre économique (le secteur d'activités, les produits et services, le marché, le bilan, la santé financière...), d'ordre social (le management, les collaborateurs, la gestion du personnel, la culture d'entreprise...), d'ordre technique (les technologies mises en œuvre, les machines, l'organisation du travail...), mais aussi d'ordre «physique» (l'implantation de l'entreprise, ses bâtiments et ses machines, son caractère plus ou moins bruyant, ses rejets dans l'atmosphère ou dans l'eau...). Pour faire court, nous acceptons l'idée que ces éléments confèrent à chaque organisation son identité propre, une singularité, qui la distingue des autres, y compris de celles qui exerceraient le même métier qu'elle.

Mais l'identité d'une organisation n'est cependant pas accessible directement comme telle, ni aux membres de cette organisation, ni aux personnes qui lui sont extérieures. Au fond, l'identité d'une personne comme celle d'une entreprise n'est accessible qu'au travers de l'image qu'elle donne d'elle-même et qui se diffuse à partir d'elle. Ainsi, en corollaire de la notion d'identité, a-t-on nécessairement celle d'image. Être-là, c'est déjà communiquer. Il y a toujours une image donnée, offerte, diffusée. Et cette image peut être définie comme un ensemble d'informations relatives à l'objet considéré.

A cette idée, en émission, d'image offerte, donnée ou « diffusée » selon le terme de G. Marion ${ }^{1}$, correspond, en réception, un concept d'image perçue ou reçue par quelqu'un qui d'une manière ou d'une autre s'est trouvé dans le champ des informations en question. Les éléments de cette réception sont particulièrement bien étudiés par la psychologie (l'attention, la perception, la compréhension, la mémorisation, l'action, par exemple) qui explique une partie des phénomènes possibles de distorsion des informations à ce niveau.

1 Marion, G. (1989), Les images de l'entreprise, Paris, Les Editions d'organisation. 
Cependant, le destinataire - qu' une vision transmissive - et donc partielle - de la communication assimile souvent à une « cible» - n'est pas seul dans un vide social. Il n'est évidemment ni passif, ni isolé. Il fait partie d'un milieu social, d'un «public».

Notre lien à la société est en effet médiatisé par notre appartenance à un milieu. Il n'y a pas l'individu d'un côté et la société de l'autre. C'est au sein du milieu social (la famille, l'école, les institutions et groupes fréquentés...) que chacun est " socialisé », que chacun apprend le cadre de référence lui permettant à la fois de se comporter à bon escient et de légitimer les comportements adoptés. Dans l'ordre des opinions, nous acquérons ainsi un système de représentations ${ }^{1}$ et de codes culturels, une sorte de « logiciel social», nous permettant d'interpréter les informations que nous recevons. Et dans l'ordre des comportements, nous apprenons à jouer les différents rôles sociaux que l'on peut attendre de quelqu'un dans différents milieux et le sien en particulier.

Par conséquent, chacun lit les informations qui lui parviennent avec en arrière-plan son système d'interprétation, son «logiciel» de traitement des données ancré en lui par sa socialisation. On doit donc s'attendre à ce que les informations perçues ou reçues individuellement soient interprétées en fonction des représentations sociales propres aux milieux sociaux concernés.

Si l'image reçue renvoie à l'aspect individuel de la réception, le concept de « réputation » (ou encore celui « d'image déposée », proposé par Gilles Marion) renvoie quant à lui au résultat de ce «mixage » incontournable entre l'image reçue et son interprétation au travers du filtre des représentations sociales de celui qui les reçoit. La réputation (ou «image déposée ») est ainsi l'opinion partagée par les membres d'un milieu ou d'un public au sens collectif du terme. De ce point de vue, les représentations sociales et les codes culturels forment un système de traitement de l'information conduisant à la construction de cette « image déposée », de cette « réputation».

Rappelons que jusqu'ici, l'ensemble du processus décrit n'implique en rien l'existence de «communicateurs » (fig. 1). L'organisation est

1 Jodelet, D. (1989), Les représentations sociales, Paris, PUF. 
un fait social. Au sens même de la pragmatique de la communication : «On ne communique pas. On prend part à une communication» (R. Birdwhistell $)^{1}$.

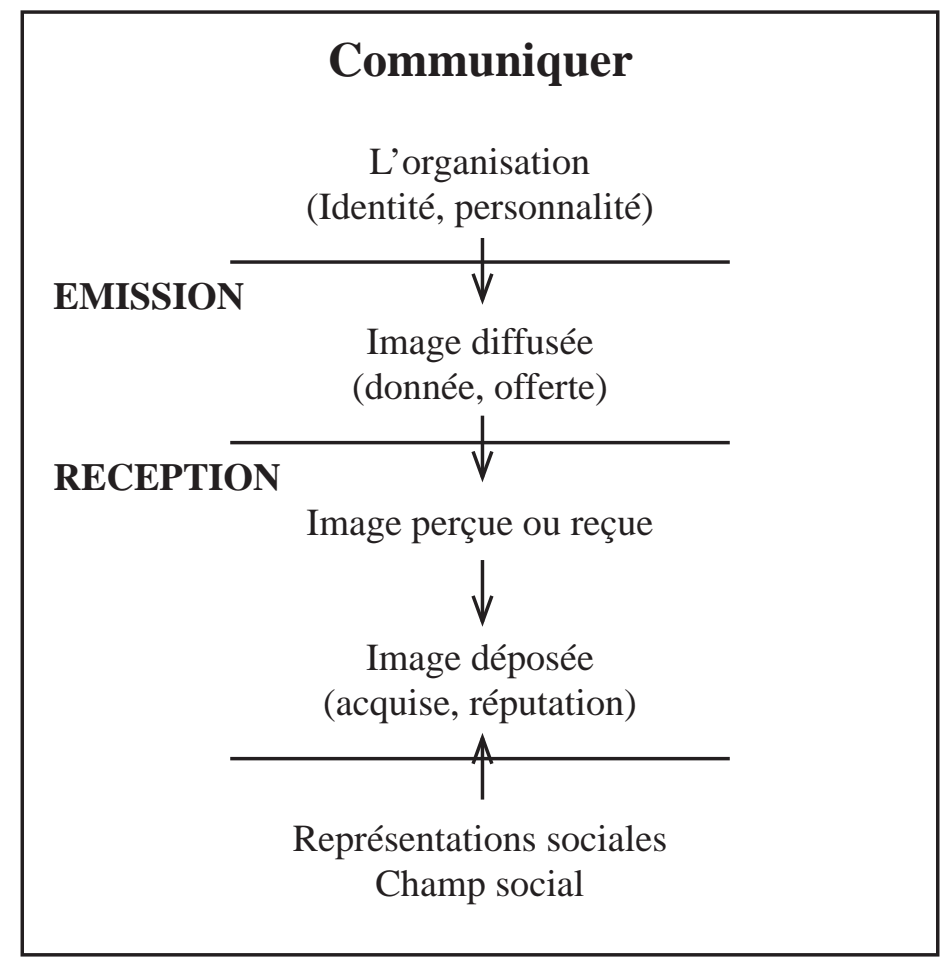

\section{« Faire de la communication »}

Devant la situation que nous venons de décrire et les enjeux qu'elle recouvre, les organisations (pour ne parler que d'elles ici, mais il pourrait bien sûr s'agir aussi d'un gouvernement, d'une personnalité publique, d'un parti politique, d'une collectivité, etc) vont évidemment chercher à maîtriser et à influencer l'information fournie à leur propos, à «gérer leur image », à manager leur communication. L'organisation - en réalité, sa direction - va donc développer (ou faire développer), vers ses membres comme vers ses publics externes, des politiques de

1 Birdwhistell, R., cité par Yves Winkin (1981), La nouvelle communication, Paris, Seuil, p.75. 
communication. Les « communicateurs » entrent en scène. La gestion de l'image, comme plus largement celle de l'information, se professionnalise.

Il ne s'agit pas en effet de laisser les choses au hasard et de risquer ainsi des conséquences pénalisantes pour l'organisation, ses produits ou ses services, d'autant moins aujourd'hui dans un univers considérablement médiatisé et globalisé. D'où sans doute l'explosion relativement récente de ces «métiers de la communication » (certains sont néanmoins anciens) et des stratégies de communication qui leur sont liées : services d'information, services de presse, de marketing, de publicité, de relations publiques, de médiation, de mécénat, de sponsoring ou de lobbying...

Pour mener une stratégie de communication cohérente, d'une part l'organisation va réfléchir à sa propre réalité, à sa propre identité, afin de déterminer l'image qu'elle pense avoir d'elle-même («image de soi », « prisme de l'identité » selon Kapferer ${ }^{1}$ ). Elle va aussi prendre connaissance et conscience de l'image qu'elle a auprès de ses publics les plus significatifs. Enfin, après ce double diagnostic, elle va s'efforcer de définir une stratégie qui se déclinera sans doute selon un «plan de communication » intégré (d'information, de publicité, de relations publiques, d'événements, de crise...) en y associant des indicateurs de performance dans le temps pour en permettre le pilotage fin et l'évaluation (Libaert et de Marco) ${ }^{2}$.

Fondamentalement, le « communicateur» est ainsi au centre d'un dispositif en double boucle de feedback débouchant sur des initiatives visant à rapprocher et à optimiser « image recherchée » et « réputation » (fig. 2). D'où aussi, notons-le au passage, l'importance des métiers du « retour d'information » et du diagnostic (sondages, études d'opinion ou d'image, bench marking, revue et analyse de presse, marketing stratégique, analyse des ventes, audits, etc)

1 Kapferer, J.N. (1988), «Maîtriser l'image de l'entreprise : le prisme de l'identité, in Revue Française de gestion», 71, pp76-82.

2 Libaert, Th. et de Marco, A. (2006), Les tableaux de bord de la communication. Indicateurs de pilotage et évaluation des résultats, Paris, Dunod. 
Aux informations diffusées spontanément par l'organisation du seul fait de son existence dans le champ social (et donc sans initiative particulière de sa part en matière de politique de communication), viennent de s'ajouter ainsi des comportements (des actes de communication) délibérés de professionnels aux objectifs et mandats variables : informer, expliquer, motiver, persuader, vendre... Il s'agit cette fois de «faire de la communication », donc, à nos yeux, d'agir sur les systèmes de comportements et d'opinions des publics concernés, agir sur la « réputation », modifier ou consolider « l'image déposée».

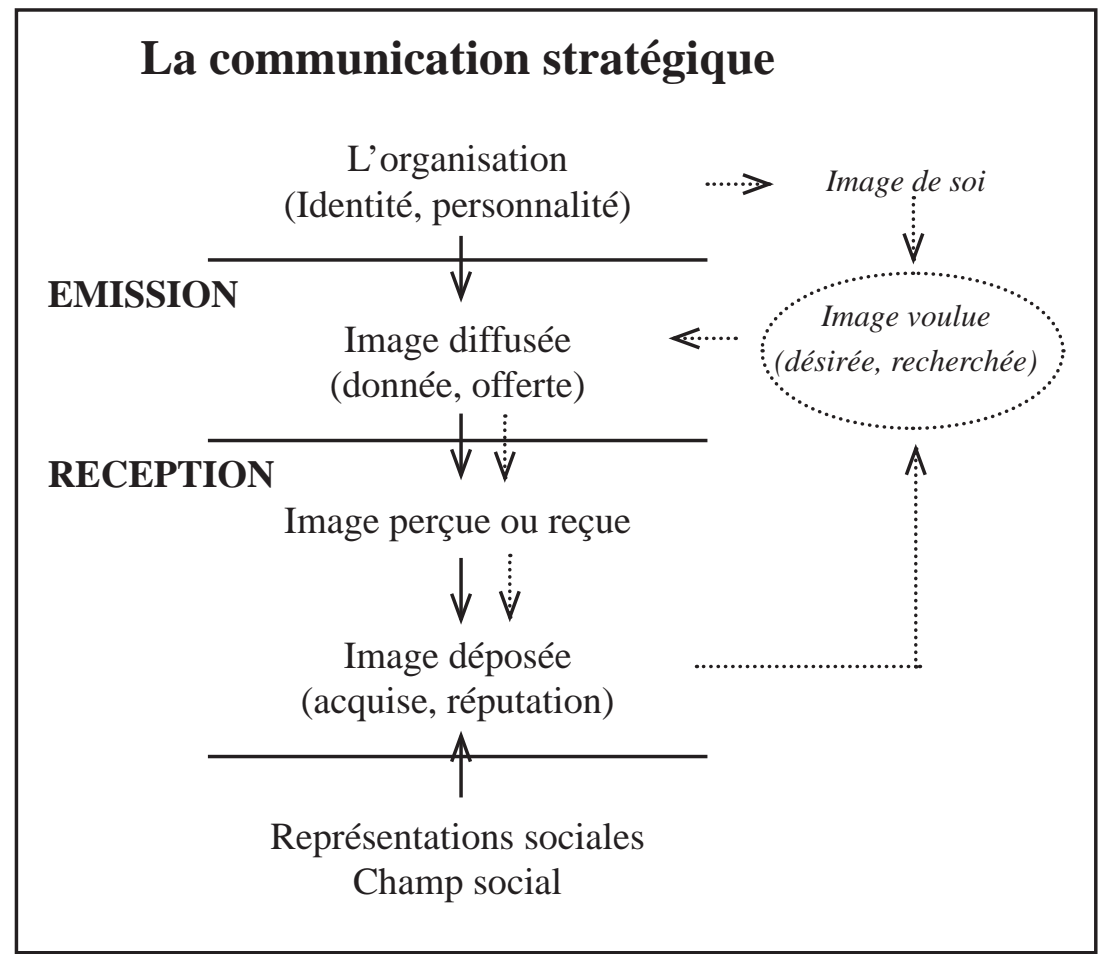
bles :

A cet égard, $\mathrm{P}$. Moliner ${ }^{1}$ suggère trois modalités d'actions possi-

1 Moliner, P., (1996), Images et représentations, Grenoble, Presses Universitaires de Grenoble. 
- diffuser de l'information (ou l'améliorer). On pourrait penser à l'information, aux relations de presse ou à la publicité, par exemple.

- modifier le «cadrage » servant de référence au classement des informations par le public concerné (modifier la représentation utilisée, inciter à en prendre une autre) ; les relations publiques, le sponsoring, le mécénat ou la communication de crise peuvent aller dans ce sens.

- changer les représentations elles-mêmes, ce qui serait à ses yeux le plus difficile ; la propagande, la désinformation par l'organisation délibérée de rumeurs, le «lynchage médiatique », certaines créations d'événements pourraient relever de ce point de vue.

\section{Un espace public}

A ces deux flux d'information organisationnels (l'un spontané et l'autre au moins partiellement maîtrisé), il faut évidemment en ajouter un troisième : les organisations ne sont généralement pas seules à parler d'elles, de leurs produits ou de leurs services. D'autres acteurs sont légitimement parties prenantes au débat : les pouvoirs publics, les interlocuteurs sociaux, les associations de consommateurs ou de citoyens, les concurrents, des experts et bien sûr la presse relayant les uns et les autres ou agissant d'initiative (fig.3).

$\mathrm{Si}$ au sein des organisations certains rêvent parfois de voir les journalistes fonctionner comme des relais de leur propre conception des choses, ils doivent bien vite constater qu'il n'en est régulièrement rien. Le métier du journaliste est d'informer. Il n'est pas celui du publicitaire ou du porte-parole.

L'organisation profite des médias (par exemple lorsqu'un événement favorable est rapporté ou qu'elle loue un espace publicitaire). Mais les médias peuvent aussi se révéler bien gênants pour l'organisation (par exemple lorsqu'ils couvrent un conflit ou une crise qui l'affecte). Pour l'organisation, «faire - ou ne pas faire - de la communication » est bien un risque à prendre dans un espace public pluriel où la moindre contradiction peut avoir pour elle des conséquences redoutables. D' autant plus que les publics, comme on l'a dit, feront de toute manière leur propre 
lecture des informations diffusées par les différentes sources auxquelles ils auront eu accès.

Toute «stratégie de communication » (qu'il s'agisse d'information, y compris la presse d'entreprise, de publicité, de relations publiques, etc.) est donc en fait une intervention psychosociologique directe ou indirecte, mais délibérée, sur le système d'opinion et de comportements d'une société ou d'une partie de la société en vue d'influencer dans un sens qui soit favorable à ses initiateurs.

La théorie de la communication «à deux étages » (Katz et Lazarsfeld ${ }^{1}$; Westley et Mc Lean) ${ }^{2}$ et son prolongement dans la théorie de la « mise à l'agenda » (Mc Combs et Shaw; Mc Combs) ${ }^{3}$ expliquent bien les phénomènes qui viennent d'être présentés. Ces théories n'envisagent toutefois pas comme tel le cas des organisations et ne font pas le lien avec la théorie de la pragmatique de la communication.

Pour ces théories, lorsqu'une information est diffusée ou transmise, son influence effective va dépendre largement de l'appropriation qui en sera faite au travers des appartenances sociales où interviennent des « leaders d'opinion », des « influenceurs », des « avocats » plaidant pour certaines causes plutôt que d'autres . La « mise à l'agenda » d'informations n'implique pas une influence linéaire sur l'opinion ou le comportement qu'un public peut avoir. Il y a donc deux temps à identifier : l'information diffusée et l'élaboration d'une opinion « légitime » qui se noue au sein du milieu social .

De ce point de vue, il y a donc une triple dimension à prendre en compte dans l'analyse : celle de l'élaboration de l'agenda médiatique (agenda building), celle de la mise à l'agenda de sujets dans la société par les médias (agenda setting) et celle de l'émergence d'opinions propres aux publics concernés par les informations ainsi mises à l'ordre du jour.

1 Katz, E.et Lazarsfeld, P. (1955), Personal influence, New-York, The Free Press of Glencoe et Lazarsfeld, P., Berelson, B.,et Gaudet, H., (1948), The people's choice, New-York, Columbia University Press.

2 Westley et Mac Lean, (1957), A conceptual model for mass communication research, in Journalism quaterly, University of Minesota, 34, pp31-38.

3 McCombs, M. et Shaw, D.L (1972), The agenda-setting function of mass-media, in Public Opinion Quaterly, 36, pp.176-187 et McCombs, M. (2004), Setting the agenda. The mass media and public opinion, Cambridge, Polity Press. 


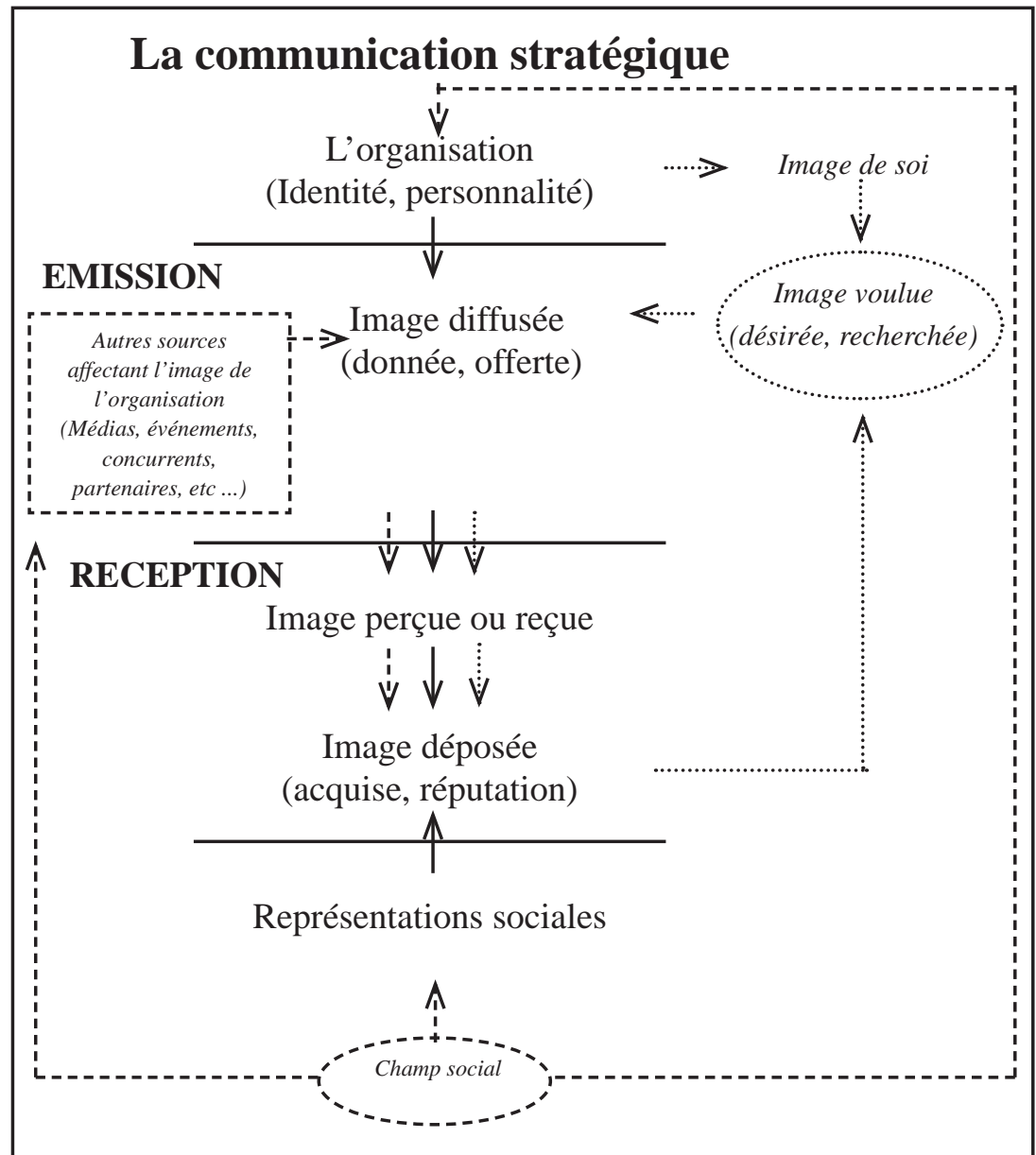

\section{Communication et légitimation : le « communicateur-symptôme »}

Ceci nous permet de revenir à notre question de départ : que nous révèlent donc les pratiques professionnelles des métiers de la communication quant à la nature des processus de légitimation mis en œuvre par les organisations?

On peut admettre que la dynamique d'une société est le produit des rapports entre des milieux sociaux qui à la fois coopèrent et entrent en conflit pour déterminer un ordre social légitime s'imposant à tous à un moment donné de l'histoire (la culture, l'idéologie dominante, la loi, la règle, les valeurs, les normes, etc.). On comprend alors que les différents milieux 
sociaux cherchent à imposer leur interprétation comme étant la plus légitime et comme étant celle qui doit s'imposer sinon à tous, du moins au plus grand nombre. La notion de « culture dominante » n'exprime rien d'autre.

Dans cet univers social nécessairement multiple, l'enjeu de l'action des communicateurs, entre le pouvoir qui les mandate et les publics visés ou concernés, est ainsi clairement de parvenir à maintenir ou à implanter «l'ordre des choses", le «sens commun», le « discours légitime » souhaité par l'organisation sans donner à voir qu'il exprime ou résulte en fait d'un rapport de force social (voir P. Bourdieu et le concept de « violence symbolique » par adhésion ou imposition) ${ }^{1}$.

De ce point de vue, on peut penser que le métier de communicateur est inscrit d'emblée dans une sorte de paradoxe de base (en quelque sorte ontologique) : devoir dévoiler (faire connaître, éclairer le projet organisationnel ou ses produits) et occulter en même temps (ne pas donner à voir le rapport de forces social sous-jacent à ce qu'on doit faire connaître). Il n'est pas étonnant dès lors que ce métier reste en définitive peu visible ou cherche à rester dans l'ombre (un « spin doctor » pourrait-il s'afficher comme tel ?). Le communicateur s'efface (relativement) devant l'organisation, le personnage ou la cause qu'il met en valeur.

Les processus de légitimation que les communicateurs conduisent renvoient donc à la vision que l'organisation (le management, les propriétaires, le pouvoir en place) a, d'une part, du lien social à valoriser au sein de l'organisation (sur le versant « communication interne ») ${ }^{2}$ et, d'autre part, de son propre rôle dans la société (sur le versant « externe »). Quelle est donc au juste la signification de cette notion de « responsabilité sociale de l'entreprise » si souvent mise en avant?

Si l'intervention des professionnels de la communication va dans le sens de l'occultation des rapports de forces ou de la diversité des enjeux propres aux différents acteurs concernés, elle légitime ces rapports de force. C'est sans doute en filigrane porter le rêve ou l'utopie d'une pensée unique en organisation comme dans la société. Cette ligne

1 Bourdieu, P. (1996), Sur la télévision, Suivi de l'emprise du journalisme, Paris, Liber et Olivesi, S. ( 2005), La communication selon Bourdieu, Paris, L'Harmattan.

2 de Saint-Georges, P. (1993), «Culture d'entreprise, communication interne et stratégies de changement», in Communication et organisation, 4, Bordeaux. 
de conduite a cependant ses fervents adeptes, sans doute héritiers en la matière du « one best way » de jadis.

Si l'action des communicateurs s'inscrit dans une logique du dévoilement des enjeux, de la pluralité des opinions et de la complexité des conduites, elle valorise alors un lien social pluriel. Dans cette hypothèse, c'est la diversité, fondement même de la société, qui est mise au service de la performance organisationnelle ou sociale. L'option est radicalement différente.

Autrement dit, on doit soutenir l'idée que le traitement du paradoxe « ontologique » du communicateur (dévoiler et occulter), tel qu'il est concrètement mis en œuvre au travers des pratiques professionnelles observables à un moment dans une organisation donnée ${ }^{1}$, est le révélateur essentiel de l'objet et du système de légitimation mis en place. Et il serait de ce fait particulièrement intéressant de multiplier les études empiriques sur les « épreuves au travail » vécues par les communicateurs dans leurs pratiques quotidiennes dans l'une et l'autre des deux logiques d'action que nous venons de cerner ${ }^{2}$.

D'un point de vue éthique et politique, l'action des communicateurs (et de ceux qui les mandatent) posera problème chaque fois qu' elle aura le projet délibéré d'imposer une orthodoxie (ou de valoriser l'adhésion à une orthodoxie) en occultant les enjeux qui la sous-tendent. Il n'y a pas d'alternative à la pluralité dans une société démocratique.

1 DE SAINT-GEORGES, P. (2000), «Les fils rouges du journalisme d'entreprise», in 1950-2000, Cinquante ans de Presse d'entreprise en Belgique, Bruxelles, Association Belge de la Presse d'Entreprise (ABPE), pp 38 à 62.

2 PÉRILLEUX, Th. (2001), Les tensions de la flexibilité. L'épreuve du travail contemporain, Paris, Desclée de Brouwer. 